Article

\title{
Three-Parameter Interval Grey Number Multi-Attribute Decision Making Method Based on Information Entropy
}

\author{
Sha Fu \\ Department of Information Management, Hunan University of Finance and Economics, No.139, Section 2, \\ Fenglin Road, Yuelu District, Changsha 410205, China; fusha15@163.com; Tel.: +86-138-7598-2792 \\ Academic Editor: Mehmet Pakdemirli \\ Received: 8 October 2015; Accepted: 5 May 2016; Published: 11 May 2016
}

\begin{abstract}
Aimed at the situation that the plan attribute value is a three-parameter interval grey number and that attribute weight is uncertain, a three-parameter interval grey number multi-attribute decision making method based on information entropy is proposed. In this study, we combine the three-parameter interval grey number decision-making problem with information entropy, build the distance entropy model of the three-parameter interval grey number, and provide a solution to the empowerment problem of the three-parameter interval grey number in grey decision-making problems. On this basis, we build the uncertainty decision making framework based on Technique for Order Preference by Similarity to Ideal Solution (TOPSIS) thinking and obtain the comprehensive distances of plans from positive and negative ideal solutions through calculation. We thus determine the closeness, and, based on this value, organize these plans. Finally, we verify the feasibility and effectiveness of the proposed methods through case analysis.
\end{abstract}

Keywords: information entropy; three-parameter interval grey number; grey distance entropy; multi-attribute decision making; closeness

\section{Introduction}

Multi-attribute decision making is an important part of modern scientific decision-making, and its theories and methods have been widely applied in engineering design, social life, economic system and management science and other fields. The multi-attribute decision making problem has profound theoretical significance and wide practical application background in several fields. Its study has always been an important topic of general interest. In real life, due to the complexity and uncertainty of objective things as well as ambiguity of human thinking, people rarely give exact values to the evaluation of things in decision making process.

In the actual decision-making process, much information has uncertainty and ambiguity, so decision-makers hardly use exact values to describe them in the evaluation process. As a means to deal with multi-attribute uncertainty decision making problem, interval grey number [1], has been considered by many scholars since it was proposed by Professor Deng Julong. Interval grey numbers are usually the upper and lower limits of the range, and the evaluation opportunities of numbers between upper and lower limits are considered equal. However, the evaluation opportunities of grey numbers in the range are unequal. Thus, when integrating the effect evaluation values of plans, the calculation results will further expand the value range of interval grey number, thus resulting in larger errors. Aimed at insufficiencies of interval grey number, Luo [2] introduced the three-parameter interval grey number, which extended multi-attribute decision-making to the situation that decision-making information is the three-parameter interval grey number, and discussed the decision-making problem assuming that the plan index value was the interval grey number 
and the maximum grey number evaluation possibility is known. Domestic and foreign literatures show that many experts and scholars have conducted theoretical research on the three-parameter interval grey number from different angles, and they have obtained certain research achievements [3]. For example, G.R. Jahanshahloo et al. [4] presented a new TOPSIS (Technique for Order Preference by Similarity to Ideal Solution) method for ranking DMUs (Decision Making Unit) with interval data yielding the interval score for each alternative, and in the end we show that data is deterministic. Serkan Genç et al. [5] introduced the concept of interval multiplicative transitivity of an interval fuzzy preference relation and showed that, by solving numerical examples, the test of consistency and the weights derived by the simple formulas based on the interval multiplicative transitivity produce the same results as those of linear programming models. Mohammad Hassan Kamfiroozi et al. [6] proposed the application of a new multi-attribute decision-making model for enterprise resource planning selection based on Balanced Score Card. Lan and Fan [7] gave the fuzzy value of three-parameter interval value, definition of fuzzy set of three-parameter interval value and calculation relationship, defined a distance on the fuzzy value of three-parameter interval value and applied it in the decision-making analysis based on fuzzy set information of three-parameter interval value. Wang and Liu [8] defined the concept of relative dominance degree in combination with a three-parameter interval grey number and real number, and proposed an ordering method of three-parameter interval grey number based on relative dominance degree. Wang and Dang [9] proposed a dynamic multi-attribute decision-making method based on prospect theory aimed at the situation that decision-making information is the three-parameter interval grey number. Yan et al. [10] considered the impact of decision-makers' index satisfaction domain and risk attitude on group decision-making, and proposed the three-parameter interval grey number group grey target decision-making method based on prospect theory. Current research may have some defects, for example in reference [7], the proposed formula for calculating the distance between three-parameter interval-valued fuzzy values is still the formula based on geometrical distance, which is of insufficient discriminability. In reference [8], the distance measure and sorting method for three-parameter interval grey number cannot reflect the essential value characteristics of three-parameter interval grey number.

Basically, the three-parameter interval grey number refers to the known interval grey number of gravity-center with the maximum possibility of value-given, i.e., three-parameter interval grey number can be expressed as $a(\otimes) \in\left[a^{l}, a^{*}, a^{u}\right]\left(a^{l} \leqslant a^{*} \leqslant a^{u}\right)$, where $a^{*}$ is the value that $a(\otimes)$ is the most likely to be, and gravity center is unknown, i.e., the so-called interval grey number. Using the three-parameter interval grey number to perform evaluation can guarantee the value range of interval grey number, highlight the gravity center point with the maximum possibility of value-given, and cover the shortage of grey number "poor" information, making the evaluation results more accordant with practical engineering. The research contributions described above provide some ideas for solving the three-parameter interval grey number decision-making problem. However, it is notable that there is less research on the multi-attribute decision making problem to show that decision making information is s three-parameter interval grey number and attribute weight is uncertain. In this study, based on current research situation, we propose corresponding decision-making methods and build the grey distance entropy model to meet the needs of such decisions-making.

\section{Preliminaries}

\subsection{Interval Grey Number}

In grey system theory [11], a number, of which the approximate range is known while the accurate value is unknown, is called a grey number. The grey number is the basic unit of a grey system. Suppose $a(\otimes) \in\left[a^{L}, a^{U}\right], a^{L}<a^{U}$, the grey number with lower limit value $a^{L}$ and upper limit value $a^{U}$ is called the interval grey number, $a(\otimes)$ is an interval grey number, if $a^{L}=a^{U}, a(\otimes)$ can be degraded into a real number. 


\subsection{Three-Parameter Interval Grey Number}

Definition 1. Set the grey number $a(\otimes)=\left[a^{l}, a^{*}, a^{u}\right]$ as a three-parameter interval grey number. Wherein, $a^{l} \leqslant a^{*} \leqslant a^{u}, a^{l}$, $a^{u}$ are respectively the lower and upper limits of the range. $a^{*}$ is the number of maximum evaluation possibility in this range, which is called the focus of interval grey number. When $a^{l}=a^{*}=a^{u}$, the three-parameter interval grey number degenerates into a real number. When two of $a^{l}, a^{*}$ and $a^{u}$ are the same, the three-parameter interval grey number degenerates into an interval grey number.

Definition 2. Set $a(\otimes)=\left[a^{l}, a^{*}, a^{u}\right], b(\otimes)=\left[b^{l}, b^{*}, b^{u}\right]$, then:

(1) $a(\otimes)+b(\otimes)=\left[a^{l}+b^{l}, a^{*}+b^{*}, a^{u}+b^{u}\right]$;

(2) $\quad a(\otimes)-b(\otimes)=\left[a^{l}-b^{u}, a^{*}-b^{*}, a^{u}-b^{l}\right]$;

$$
\lambda a(\otimes)=\left[\lambda a^{l}, \lambda a^{*}, \lambda a^{u}\right], \lambda>0
$$

Definition 3. Set $a(\otimes)=\left[a^{l}, a^{*}, a^{u}\right], b(\otimes)=\left[b^{l}, b^{*}, b^{u}\right]$, then say:

$$
d(a(\otimes), b(\otimes))=\alpha\left|a^{l}-b^{l}\right|+\beta\left|a^{*}-b^{*}\right|+(1-\alpha-\beta)\left|a^{u}-b^{u}\right|
$$

is the distance between $a(\otimes)$ and $b(\otimes)$. Wherein, $0 \leqslant \alpha<0.5,0.5 \leqslant \beta \leqslant 1$. In general, we can take $\alpha=0.25, \beta=0.5$.

Definition 4. Set grey number $a(\otimes)=\left[a^{l}, a^{*}, a^{u}\right], b(\otimes)=\left[b^{l}, b^{*}, b^{u}\right]$, when

$$
a^{*}+\frac{a^{u}+a^{l}}{2}>b^{*}+\frac{b^{u}+b^{l}}{2}
$$

say $a(\otimes)>b(\otimes)$.

\subsection{Information Entropy and Grey Distance Entropy}

The concept of entropy was first proposed by the German physicist Rudolf Clausius in 1865. After that, in 1948, the father of information theory C.E.Shannon introduced the concept of entropy in the field of information as a measure to the degree of information disorder [12]. Shannon systematically led the way to measuring information $H(X)=H\left(p_{1}, p_{2}, \cdots, p_{n}\right)=-k \sum_{i=1}^{n} P_{i} \log P_{i}$, the used probability statistical method and the use of entropy to measure the uncertainty of a random event or the amount of information, which laid the scientific theoretical foundation of modern information theory, and extended the quantitative application of entropy to the relevant research on uncertainty of the system and disorder quantification [13]. Information entropy is a mathematical abstract concept, meaning that the occurrence probability of specific information is used to indicate uncertainty of information in the information system. The higher the certainty of an information system is, the lower the information entropy is; conversely, the higher the entropy is [14].

Definition 5. Set $a^{l}=a, a^{*}=m, a^{u}=b, b^{l}=c, b^{*}=n, b^{u}=d$ in $a(\otimes)$ and $b(\otimes)$, define the three-parameter interval grey number $a(\otimes)=[a, m, b](0<a \leqslant m \leqslant b)$ and $b(\otimes)=[c, n, d](0<c \leqslant$ $n \leqslant d)$, then say [15]:

$$
\begin{aligned}
& H(D)=\frac{1}{3}\left[\left(-\frac{a}{a+c} \ln \frac{a}{a+c}\right)+\left(-\frac{c}{a+c} \ln \frac{c}{a+c}\right)+\left(-\frac{b}{b+d} \ln \frac{b}{b+d}\right)\right. \\
& \left.\quad+\left(-\frac{d}{b+d} \ln \frac{d}{b+d}\right)+\left(-\frac{m}{m+n} \ln \frac{m}{m+n}\right)+\left(-\frac{n}{m+n} \ln \frac{n}{m+n}\right)\right]
\end{aligned}
$$


as the distance entropy of three-parameter interval grey number $a(\otimes)$ and $b(\otimes)$. The grey distance entropy represents the measurement of proximity of $a(\otimes)$ and $b(\otimes)$, but does not represent the distance between them.

\section{Three-Parameter Interval Grey Number Multi-Attribute Decision-Making Method}

Set a multi-attribute decision-making problem. Its plan set is $A=\left\{A_{1}, A_{2}, \cdots, A_{n}\right\}$, its attribute factor set is $C=\left\{C_{1}, C_{2}, \cdots, C_{m}\right\}$, the corresponding attribute weight is $w_{j}$ and $\zeta_{j} \leqslant w_{j} \leqslant \xi_{j}, \zeta_{j}, \xi_{j} \in$ $[0,1]$, and $\sum_{j=1}^{m} w_{j}=1 . u_{i j}(i=1,2, \cdots, n ; j=1,2, \cdots, m)$ is the effect sample value of Plan $A_{i}$ on the attribute $C_{j}$. This effect sample value is a three-parameter interval grey number, noting $u_{i j}(\otimes) \in\left[\underline{u}_{i j}, \tilde{u}_{i j}, \bar{u}_{i j}\right]$. Wherein, $0 \leqslant \underline{u}_{i j} \leqslant \tilde{u}_{i j} \leqslant \bar{u}_{i j}$. It can be concluded the decision making matrix is $U=\left(u_{i j}(\otimes)\right)_{n \times m}$.

\subsection{Normalization of Decision Making Matrix}

To eliminate the difference of plan evaluation information in dimension under different attributes and increase comparability, the grey range conversion is introduced [2].

For efficiency index value, there is:

$$
\underline{r}_{i j}=\frac{\underline{u}_{i j}-\underline{u}_{j}^{\nabla}}{\overline{\bar{u}}_{j}^{*}-\underline{u}_{j}^{\nabla}}, \quad \tilde{r}_{i j}=\frac{\tilde{u}_{i j}-\underline{u}_{j}^{\nabla}}{\bar{u}_{j}^{*}-\underline{u}_{j}^{\nabla}}, \quad \bar{r}_{i j}=\frac{\bar{u}_{i j}-\underline{u}_{j}^{\nabla}}{\bar{u}_{j}^{*}-\underline{u}_{j}^{\nabla}}
$$

For cost index value, there is:

$$
\underline{r}_{i j}=\frac{\bar{u}_{j}^{*}-\bar{u}_{i j}}{\bar{u}_{j}^{*}-\underline{u}_{j}^{\nabla}}, \quad \tilde{r}_{i j}=\frac{\bar{u}_{j}^{*}-\tilde{u}_{i j}}{\bar{u}_{j}^{*}-\underline{u}_{j}^{\nabla}}, \quad \bar{r}_{i j}=\frac{\bar{u}_{j}^{*}-\underline{u}_{i j}}{\bar{u}_{j}^{*}-\underline{u}_{j}^{\nabla}}
$$

wherein, $\bar{u}_{j}^{*}=\max _{1 \leqslant i \leqslant n}\left\{\bar{u}_{i j}\right\}, \underline{u}_{j}^{\nabla}=\min _{1 \leqslant i \leqslant n}\left\{\underline{u}_{i j}\right\}$, then $r_{i j}(\otimes) \in\left(\underline{r}_{i j}, \tilde{r}_{i j}, \bar{r}_{i j}\right)$ is the three-parameter interval grey number in the range of $[0,1]$. Thus, it can be concluded the standardized decision-making matrix is $R=\left(r_{i j}(\otimes)\right)_{n \times m}$.

\subsection{Grey Distance Entropy of Three-Parameter Interval Grey Number}

For the multi-attribute decision-making problem that the attribute value is the three-parameter interval grey number, the difference of every attribute value can be expressed by the distance entropy of three-parameter interval grey number. For attribute, $C_{j}$, if the deviation of Plan $A_{i}$ from other plans is expressed by $D_{i j}$, then it can be defined:

$$
D_{i j}=\sum_{k=1}^{r} H(D)_{\otimes i j \otimes k j} w_{j}
$$

For attribute $C_{j}, D_{j}$ represents the total grey distance entropy deviation of each plan from other plants. The grey entropy deviations of all plans under the attribute $C_{j}$ are larger, indicating that the effect of the attribute index on plan decision making and ordering is smaller; conversely, if the deviation is smaller, this indicates that the effect is larger.

$$
D_{j}=\sum_{i=1}^{r} D_{i j}=\sum_{i=1}^{r} \sum_{k=1}^{r} H(D)_{\otimes i j \otimes k j} w_{j}
$$

wherein, $k \neq i$. 
For all attributes $C_{j}$, if the distance entropy of plan to be evaluated and other decision making plans is smallest, then the attribute obtained is certainly optimal, and the empowerment model of the attribute weight is:

$$
\left\{\begin{array}{l}
\min D=\sum_{j=1}^{s} D_{j}=\sum_{j=1}^{s} \sum_{i=1}^{r} \sum_{k=1}^{r} H(D)_{\otimes i j \otimes k j} w_{j} \\
0 \leqslant a_{j} \leqslant w_{j} \leqslant b_{j} \leqslant 1 \\
\sum_{j=1}^{s} w_{j}=1 \\
i=1,2, \cdots, r ; j=1,2, \cdots, s ; k \neq i
\end{array}\right.
$$

By the Equation (7), determine the single-objective optimization model. Using software programming to solve the mathematical programming model, we obtain the attribute weight vector $w=\left(w_{1}, w_{2}, \cdots, w_{m}\right)$.

\subsection{Decision Making Method}

The steps of this decision making method are as follows:

Step 1: use grey number range conversion to standardize the decision-making matrix, and obtain the standardized three-parameter interval grey number decision-making matrix.

Step 2: Use Equations (5)-(7) to obtain the attribute weight vector.

Step 3: Determine the positive ideal solution and negative ideal solution.

Positive ideal solution $\bar{a}$ is:

$$
\left\{\begin{array}{l}
\bar{a}=\left(\bar{a}_{1}, \bar{a}_{2}, \cdots, \bar{a}_{n}\right) \\
\bar{a}_{j}=\max _{1 \leqslant i \leqslant m} a_{i j}
\end{array}\right.
$$

Negative ideal solution $\underline{a}$ is:

$$
\left\{\begin{array}{l}
\underline{a}=\left(\underline{a}_{1}, \underline{a}_{2}, \cdots, \underline{a}_{n}\right) \\
\underline{a}_{j}=\min _{1 \leqslant i \leqslant m} a_{i j}
\end{array}\right.
$$

wherein, $j=1,2, \cdots, n$.

Step 4: Use the weight vector obtained and combine the Equation (2) to obtain the comprehensive distance $S\left(A_{i}, \bar{a}\right)$ of plans $A_{i}(i=1,2, \cdots, n)$ from positive ideal solution $\bar{a}$, as well as the comprehensive distance $S\left(A_{i}, \underline{a}\right)$ [16] from negative ideal solution $\underline{a}$.

Step 5: calculate the proximity between plan $A_{i}$ and positive and negative ideal solutions, and order the plans according to the proximity value.

$$
C_{i}=\frac{S\left(A_{i}, \underline{a}\right)}{S\left(A_{i}, \bar{a}\right)+S\left(A_{i}, \underline{a}\right)}
$$

\section{Case Analysis}

Take the carrier-based aircraft selection problem in literature [2,3] as an example. Assume the main attributes affecting the carrier-based aircraft selection are maximum speed $\left(C_{1}\right)$, cross-sea free voyage $\left(C_{2}\right)$, maximum net load $\left(C_{3}\right)$, acquisition cost $\left(C_{4}\right)$, reliability $\left(C_{5}\right)$, mobility and flexibility $\left(C_{6}\right)$. All attribute weight information is known: $w=\left(w_{1}, w_{2}, w_{3}, w_{4}, w_{5}, w_{6}\right)$. In incomplete certain information, the weight range of each attribute $0.15 \leqslant w_{1} \leqslant 0.19,0.10 \leqslant w_{2} \leqslant 0.15,0.10 \leqslant w_{3} \leqslant 0.15$, $0.10 \leqslant w_{4} \leqslant 0.15,0.19 \leqslant w_{5} \leqslant 0.23,0.23 \leqslant w_{6} \leqslant 0.25$, and $\sum_{j=1}^{6} w_{j}=1$. Now there are four models available, namely plan set $A=\left(A_{1}, A_{2}, A_{3}, A_{4}\right)$. Invite experts to respectively give the judgment 
matrix. After assembly, obtain the comprehensive judgment matrix, and standardize the attribute value to obtain the standardized three-parameter interval grey number judgment matrix $R(\otimes)$ :

$$
\begin{aligned}
& R(\otimes)=\left(r_{i j}(\otimes)\right)_{4 \times 6} \\
& =\left[\begin{array}{llllll}
{[0.78,0.80,0.85]} & {[0.50,0.55,0.58]} & {[0.90,0.95,0.95]} & {[0.80,0.82,0.85]} & {[0.45,0.50,0.57]} & {[0.90,0.95,0.97]} \\
{[0.92,0.95,1.00]} & {[0.95,0.97,1.00]} & {[0.85,0.86,0.88]} & {[0.65,0.69,0.71]} & {[0.17,0.20,0.23]} & {[0.47,0.51,0.55]} \\
{[0.70,0.72,0.78]} & {[0.72,0.74,0.75]} & {[0.95,0.98,1.00]} & {[0.94,0.97,1.00]} & {[0.80,0.83,0.85]} & {[0.80,0.82,0.85]} \\
{[0.85,0.88,0.90]} & {[0.65,0.67,0.70]} & {[0.90,0.95,0.96]} & {[0.85,0.90,0.93]} & {[0.46,0.50,0.52]} & {[0.48,0.50,0.52]}
\end{array}\right]
\end{aligned}
$$

(1) Use the Equation (4) to calculate the distance entropy of four plans under attributes. The grey distance entropy results of plan $A_{1}$ under attributes are shown in Table 1.

Table 1. Grey distance entropy of plan $A_{1}$ under attributes.

\begin{tabular}{ccccc}
\hline $\boldsymbol{H}(\boldsymbol{D})$ & $\boldsymbol{A}_{\mathbf{1}}$ & $\boldsymbol{A}_{\mathbf{2}}$ & $\boldsymbol{A}_{\mathbf{3}}$ & $\boldsymbol{A}_{\mathbf{4}}$ \\
\hline$C_{1}$ & 0.6931 & 0.6897 & 0.6919 & 0.6923 \\
$C_{2}$ & 0.6931 & 0.6520 & 0.6813 & 0.6872 \\
$C_{3}$ & 0.6931 & 0.6924 & 0.6929 & 0.6931 \\
$C_{4}$ & 0.6931 & 0.6888 & 0.6898 & 0.6923 \\
$C_{5}$ & 0.6931 & 0.5952 & 0.6630 & 0.6928 \\
$C_{6}$ & 0.6931 & 0.6482 & 0.6909 & 0.6457 \\
\hline
\end{tabular}

Similarly, calculate the grey distance entropy of $A_{i}(i=2,3,4)$ under attributes. Due to space limitation, its calculation process is omitted.

(2) By Equations (5) and (6), obtain the total grey distance entropy deviation of $A_{1}$ from other plans:

$$
D_{1}=\sum_{i=1}^{4} D_{i 1}=\sum_{i=1}^{4} \sum_{k=1}^{4} H(D)_{\otimes i 1 \otimes k 1} w_{1}=8.2790 w_{1},(k \neq i)
$$

Similarly, it can be concluded:

$$
D_{2}=8.1453 w_{2}, \quad D_{3}=8.3096 w_{3}, \quad D_{4}=8.2508 w_{4}, \quad D_{5}=7.4047 w_{5}, \quad D_{6}=8.0114 w_{6}
$$

Thus, obtain:

$$
D=8.2790 w_{1}+8.1453 w_{2}+8.3096 w_{3}+8.2508 w_{4}+7.4047 w_{5}+8.0114 w_{6}
$$

By the Equation (7), the empowerment model of interval weight can be established as follows:

$$
\left\{\begin{array}{l}
\min D=8.2790 w_{1}+8.1453 w_{2}+8.3096 w_{3}+8.2508 w_{4}+7.4047 w_{5}+8.0114 w_{6} \\
0.15 \leqslant w_{1} \leqslant 0.19 \\
0.10 \leqslant w_{2} \leqslant 0.15 \\
0.10 \leqslant w_{3} \leqslant 0.15 \\
0.10 \leqslant w_{4} \leqslant 0.15 \\
0.19 \leqslant w_{5} \leqslant 0.23 \\
0.23 \leqslant w_{6} \leqslant 0.25 \\
\sum_{j=1}^{6} w_{j}=1
\end{array}\right.
$$

Solve the above single-objective optimization model to obtain the attribute weight:

$$
w_{1}=0.150, \quad w_{2}=0.149, \quad w_{3}=0.100, \quad w_{4}=0.121, \quad w_{5}=0.229, \quad w_{6}=0.250
$$


(3) Use the Equations (8) and (9) to separately obtain the positive and negative ideal solutions of decision making.

Positive ideal solution is:

$$
\begin{aligned}
& \bar{a}=([0.92,0.95,1.00],[0.95,0.97,1.00],[0.95,0.98,1.00], \\
& [0.94,0.97,1.00],[0.80,0.83,0.85],[0.90,0.95,0.97])
\end{aligned}
$$

Negative ideal solution is:

$$
\begin{aligned}
& \underline{a}=([0.70,0.72,0.78],[0.50,0.55,0.58],[0.85,0.86,0.88], \\
& [0.65,0.69,0.71],[0.17,0.20,0.23],[0.48,0.50,0.52])
\end{aligned}
$$

(4) Use the attribute weight vector obtained and combines the Equation (2) to obtain the comprehensive distance $S\left(A_{i}, \bar{a}\right)$ of plans $A_{i}$ from positive ideal solution $\bar{a}$, as well as comprehensive distance $S\left(A_{i}, \underline{a}\right)$ from negative ideal solution $\underline{a}$.

$$
\begin{aligned}
& S\left(\bar{a}, a_{1}\right)=0.1815, \quad S\left(\bar{a}, a_{2}\right)=0.2978, \quad S\left(\bar{a}, a_{3}\right)=0.0988, \quad S\left(\bar{a}, a_{4}\right)=0.2559 \\
& S\left(\underline{a}, a_{1}\right)=0.2162, \quad S\left(\underline{a}, a_{2}\right)=0.1012, \quad S\left(\underline{a}, a_{3}\right)=0.2990, \quad S\left(\underline{a}, a_{4}\right)=0.1418
\end{aligned}
$$

(5) Use the Equation (10) to determine the proximity between plans $A_{i}$ and positive and negative ideal solutions, and order the plans according to proximity value.

$$
C_{1}=0.5436, \quad C_{2}=0.2536, \quad C_{3}=0.7517, \quad C_{4}=0.3566
$$

Thus, the final ordering result is $A_{3}>A_{1}>A_{4}>A_{2}$. We can determine the optimal plan $A_{3}$. This result is consistent with the conclusion in the literature [2,3]. Its evaluation result can provide a scientific basis for the organization's decision-makers. From the specific steps and process, it can be seen that, compared with methods used in references, the calculation process of methods proposed in this study is relatively simple, which is easily promoted and applied in practice.

\section{Conclusions}

For the multi-attribute decision making problem of a three-parameter interval grey number, this study uses information entropy theory to establish the distance entropy model of a three-parameter interval grey number, verifies the relationship between the three-parameter interval grey number and grey distance entropy, and provides a new attribute empowerment method for three-parameter interval grey number multi-attribute decision making. On this basis, this study establishes the uncertainty decision-making framework based on TOPSIS thinking, orders the plans according to its value, and obtains the comprehensive distance of plans from positive and negative ideal solutions by calculating the weight vector of attributes. By using case studies, this study discusses the application of the multi-attribute decision-making method. The above decision-making attribute has a three-parameter interval grey number and the attribute weight is uncertain in the carrier-based aircraft selection. Thus, we provide a scientific and practical decision-making method for solving such decision-making problems.

Acknowledgments: This study was supported by the Hunan Province Education and Science Planning Project (No. XJK014BGD005), by the Hunan Province Philosophy and Social Science Foundation (No. 14YBA065). Supported by the construct program of the key discipline in Hunan province.

Conflicts of Interest: The author declares no conflict of interest. 


\section{References}

1. Liu, S.; Dang, Y.; Fang, Z.; Xie, N.M. Grey System Theory and Its Application; Science Press: Beijing, China, 2010; pp. 15-16.

2. Luo, D. Decision-making methods with three-parameter interval grey number. Syst. Eng. Theory Pract. 2009, 29, 124-130. [CrossRef]

3. Luo, D.; Wang, X. The multi-attribute grey target decision method for attribute value within three-parameter interval grey number. Appl. Math. Model. 2012, 36, 1957-1963. [CrossRef]

4. Jahanshahloo, G.R.; Hosseinzadeh Lotfi, F.; Davoodi, A.R. Extension of TOPSIS for decision-making problems with interval data: Interval efficiency. Math. Comput. Model. 2009, 49, 1137-1142. [CrossRef]

5. Genç, S.; Boran, F.E.; Akay, D.; Xu, Z. Interval multiplicative transitivity for consistency, missing values and priority weights of interval fuzzy preference relations. Inform. Sci. 2010, 180, 4877-4891. [CrossRef]

6. Kamfiroozi, M.H.; Aliahmadi, A.; Jafari-Eskandari, M. Application of Three Parameter Interval Grey Numbers in Enterprise Resource Planning Selection. Int. J. Inform. Secur. Syst. Manag. 2012, 1, 72-77.

7. Lan, R.; Fan, J. TOPSIS decision-making method on three parameters interval-valued fuzzy sets. Syst. Eng. Theory Pract. 2009, 29, 129-136. [CrossRef]

8. Wang, J.; Liu, S. Method of ranking three parameters interval grey numbers and its application in interval DEA model. Syst. Eng. Electron. 2011, 33, 106-109.

9. Wang, X.; Dang, Y. Dynamic multi-attribute decision-making methods with three-parameter interval grey number. Control Decis. 2015, 30, 1623-1629.

10. Yan, S.; Liu, S.; Wu, L. A group grey target decision making method with three parameter interval grey number based on prospect theory. Control Decis. 2015, 30, 105-109.

11. Deng, J. Grey Prediction an Grey Decision; Press of Huazhong University of Science \& Technology: Wuhan, China, 2002; pp. 56-61.

12. Liang, J.; Qian, Y. Information granule and entropy theory in information system. Sci. China Ser. E Inform. Sci. 2008, 38, 2048-2065. [CrossRef]

13. Ding, W.; Wang, J.; Guan, Z.; Zhu, H. Algorithm of Attribute Reduction Based on Extension Entropy of Variable Precision Thresholding in Incomplete Information System. J. Chin. Comput. Syst. 2010, 31, 2372-2376.

14. Dzazali, S.; Zolait, A. Assessment of information security maturity: An exploration study of Malaysian public service organizations. J. Syst. Inform. Technol. 2012, 14, 23-57. [CrossRef]

15. Liu, W.; Ge, S.; Wang, N.; Yin, J. Measuring the complexity of information system based on the data complexity. Syst. Eng. Theory Pract. 2013, 33, 3198-3208.

16. Yan, S.L.; Liu, S.F.; Zhu, J.J.; Fang, Z.G.; Liu, J. TOPSIS Decision-making Method with Three-Parameter Interval Number Based on Entropy Measure. Chin. J. Manag. Sci. 2013, 21, 145-151.

(C) 2016 by the author; licensee MDPI, Basel, Switzerland. This article is an open access article distributed under the terms and conditions of the Creative Commons Attribution (CC-BY) license (http://creativecommons.org/licenses/by/4.0/). 